\title{
Estadificación clínica como factor pronóstico en cáncer de vejiga músculo-invasor
}

\section{Clinical Staging as Prognostic Factor in Muscle-Invasive Bladder Cancer}

\author{
Ana Paola Orozco-López ${ }^{1}$ David Moreno-Mesa ${ }^{1}{ }^{10}$ Juan Pablo Saldarriaga-Botero ${ }^{2}$ Jenny García-Valencia ${ }^{3}$ \\ ${ }^{1}$ Residente de urología de la Universidad de Antioquia, Medellín, \\ Colombia \\ 2 Urólogo, IPS universitaria, Universidad de Antioquia, Medellín, \\ Address for correspondence David Moreno Mesa, MD, IPS \\ Universitaria Clínica León XIII, bloque 1, piso 4 / Calle 69 \# 51c- 24 /, \\ Colombia \\ 3 Psiquiatra, PhD Epidemiología, docente de la facultad de Medicina, \\ Universidad de Antioquia, Medellín, Colombia
}

Urol Colomb 2021;30:117-122.

\section{Resumen \\ Palabras clave \\ - Neoplasias de la vejiga urinaria \\ - estadificación de neoplasias \\ - técnicas de diagnóstico urológico \\ - diagnóstico por imagen}

Abstract

Keywords
- urinary bladder
neoplasms
- neoplasm staging
- diagnostic imaging

El cáncer de vejiga músculo invasor (CV-MI), requiere de una adecuada estadificación clínica y patológica, ya que eso determina las estrategias terapéuticas y el pronóstico de la enfermedad, caracterizada por una alta morbimortalidad. Este articulo tiene como objetivo realizar una revisión de la literatura sobre la exactitud diagnóstica de las técnicas de imagen disponibles para la estadificación clínica de los pacientes con CV-MI y cómo se debería realizar el seguimiento radiológico en los pacientes llevados a cirugía. Para eso se realizó una búsqueda en la base de datos Pubmed de artículos en inglés y en español, se incluyeron artículos de revisión, metanálisis, guías clínicas, estudios retrospectivos y prospectivos de pronóstico y diagnóstico desde el año 1988 hasta el año 2020.

Muscle invasive bladder cancer (CV-MI) requires an adequate clinical and pathological staging, as this determines the therapeutic strategies and prognosis of the disease, characterized by high morbidity and mortality. This article aims to carry out a review of the literature on the diagnostic accuracy of the available imaging techniques for the clinical staging of patients with CV-MI and how the radiological follow up should be made in the patients who underwent surgical management of the disease. For this, a search was carried out in the Pubmed database of articles in English and Spanish; review articles, meta-analyzes, clinical guidelines, retrospective and prospective prognosis and diagnosis studies from 1988 to 2020 were included received

May 4, 2020

accepted

September 15, 2020

published online

February 4, 2021
DOI https://doi.org/

10.1055/s-0040-1721326.

ISSN 0120-789X.

e ISSN 2027-0119.
(C) 2021. Sociedad Colombiana de Urología. All rights reserved. This is an open access article published by Thieme under the terms of the Creative Commons Attribution-NonDerivative-NonCommercial-License, permitting copying and reproduction so long as the original work is given appropriate credit. Contents may not be used for commercial purposes, or adapted, remixed, transformed or built upon. (https://creativecommons.org/ licenses/by-nc-nd/4.0/)

Thieme Revinter Publicações Ltda., Rua do Matoso 170, Rio de Janeiro, RJ, CEP 20270-135, Brazil 


\section{Introducción}

El Cáncer de Vejiga (CV) es el más frecuente dentro de los tumores de tracto urinario ${ }^{1}$ y el séptimo cáncer diagnosticado en la población masculina en todo el mundo, con una razón hombre: mujer que varía entre $2: 1$ y 6:1 en diferentes regiones del mundo. ${ }^{2}$ Según GLOBOCAN ${ }^{3}$ para el año 2018 en Colombia se encontró una incidencia de 3,6 por cada 100.000 personasaño y una mortalidad de 1,4 por cada 100.000 personas-año.

Aproximadamente el $75 \%$ de los pacientes diagnosticados con $\mathrm{CV}$ se presentan con lesiones no músculo-invasivas (NMI) con un menor riesgo de mortalidad específica por cáncer en comparación con los tumores músculo invasores (MI) (T2 a T4) $)^{2,4}$ que corresponden al $20 \%$ a $30 \%$; cada una con comportamiento biológico, pronóstico y tratamiento diferentes. $^{5}$

Los pacientes con CV-MI tienen peores deselaces dentro del espectro de la enfermedad, con estudios clásicos que reportan mortalidad de hasta el $85 \%$ a los 2 años del diagnóstico cuando no se realiza ningún tipo de terapia ${ }^{6}$; por ello es importante determinar adecuadamente el pronóstico en tales pacientes mediante la estadificación clínica tumoral, ganglionar y patológica.

En cuanto a la estadificación clínica, actualmente las diferentes guías recomiendan emplear de manera rutinaria la Tomografía Computarizada (TAC) para el estadiaje prequirúrgico del $\mathrm{CV}$ infiltrante como técnica de imagen inicial, por su disponibilidad y razonable seguridad en el diagnóstico global. ${ }^{7}$ Sin embargo, varios estudios ponen en duda su uso rutinario en la estadificación clínica debido a su baja sensibilidad $\mathrm{y}$ especificidad, con importantes limitaciones en precisión diagnóstica.

Teniendo en cuenta que la estadificación clínica tumoral y ganglionar definirá las decisiones terapéuticas para el paciente, nos propusimos realizar una revisión de la literatura sobre la exactitud diagnóstica de las técnicas disponibles, lo que muestra una mejor relación costo efectividad para la estadificación clínica y el pronóstico que implica.

\section{Estadificación clínica por imágenes}

Tanto los CV-MI como los CV-NMI de alto grado requieren una estadificación clínica con técnicas de imagen que ayuden a valorar múltiples características que finalmente determinarán cuál es el tratamiento adecuado y el pronóstico de la enfermedad. Dentro de ellas se incluyen: la invasión local, multifocalidad vesical, compromiso de Tracto Urinario Superior (TUS), afectación ganglionar y metástasis a distancia. ${ }^{1}$

El estadiaje clínico con urografía, ecografía y Resección Transuretral (RTU) es poco fiable, con tasas de error diagnóstico del 30-50\%, sobre todo en tumores localmente avanzados, en los que es difícil establecer la afectación extravesical. Por lo cual actualmente las diferentes guías clínicas recomiendan emplear para el estadiaje prequirúrgico del $\mathrm{CV}$ infiltrante, la TAC tóracoabdomino-pélvica con fase urográfica para la valoración del
TUS, por su disponibilidad y razonable seguridad en el diagnóstico global. $^{7}$

1- UROGRAFÍA EXCRETORA: Tradicionalmente ha sido utilizada para excluir tumores sincrónicos en el TUS en pacientes con $\mathrm{CV}$; sin embargo, en la actualidad ha sido sustituida por la urotomografía y la RM. La sensibilidad en la detección de CV es baja y variable (26 al 86\%). ${ }^{8}$

2- ECOGRAFÍA: La Ecografía Convencional (US) es el método tradicionalmente empleado en el estudio del paciente con hematuria, sin embargo, presenta importantes limitaciones en el diagnóstico de infiltración de la pared vesical y de la afectación ganglionar, por lo que no es útil en la estadificación tumoral local. ${ }^{1}$ Se reporta una sensibilidad del 63\% y especificidad del $99 \%$ en la detección de $\mathrm{CV}^{9}$

La incorporación de contrastes ecográficos (CUS), permite distinguir las capas de la pared vesical y el tejido tumoral, ${ }^{1}$ lo cual aumenta la seguridad diagnóstica, con una sensibilidad de $65 \%$ frente a $60 \%$ en la US, y una especificidad de $88 \%$ frente $72 \%$ respectivamente. ${ }^{10} \mathrm{Su}$ utilización en la estadificación local parece prometedora; aunque se necesitan más estudios que lo confirmen. Está avalado por la Federación Europea de Sociedades Médicas de Ultrasonido con el fin de diferenciar entre lesiones tumorales y coágulos (LE 2 b). ${ }^{1}$

3- TOMOGRAFÍA COMPUTARIZADA MULTIDETECTOR (TCMD): El protocolo de estudio más empleado es la TAC con fase urográfica (Urotomografía) que permite una mejor valoración del TUS, ${ }^{1}$ importante para identificar posibles tumores sincrónicos presentes en el 2\% de los pacientes con $\mathrm{CV}$ y en el seguimiento posterior al tratamiento para identificar tumores metacrónicos en el TUS (3,9\%)., 11,12

En el diagnóstico primario o de recurrencia del CV, la Urotomografía presenta una sensibilidad y especificidad del 93\% y 99\% respectivamente, con un VPP del 95\%. ${ }^{13}$ La sensibilidad disminuye de forma significativa (20\%) en tumores menores de $5 \mathrm{~mm}$, y cuando se realiza precozmente tras la RTU o los tratamientos radioquimioterápicos locales; ya que provocan una inflamación reactiva local que enmascara los hallazgos. ${ }^{1,14-16}$

4- TOMOGRAFÍA POR EMISIÓN DE POSITRONES (PET): La PET es un estudio funcional que permite la valoración y cuantificación del metabolismo tisular, mediante el empleo de un radiotrazador (el más frecuente: 18F-fluordeoxiglucosa) aumentado en las lesiones tumorales. Los cambios en el metabolismo glucídico de las lesiones se dan antes que los morfológicos, lo que lleva a un diagnóstico precoz. La localización espacial de las lesiones hipermetabólicas detectadas con la PET está limitada por la poca resolución espacial de la técnica. ${ }^{1}$

La combinación del PET con estudio tomográfico (PET-CT) permite detectar ganglios metastásicos con tamaño inferior a $1 \mathrm{~cm}$ y visualización de metástasis extrapélvicas con una sensibilidad global superior a la TAC, $54 \%$ vs $41 \%$ y especificidad del $97 \%$. Sin embargo, no es útil en el estudio de metástasis cerebrales por su alto metabolismo glucídico que interfiere con la visualización. La PET-CT presenta, en la estadificación global, un 20-40\% más de hallazgos que la TAC, lo que puede suponer un cambio en el manejo terapéutico del 
paciente en el $13-27 \%$ de los casos, aunque a expensas de un sobrediagnóstico del $20 \%{ }^{1}$

5- RESONANCIA MAGNÉTICA (RM): La ventaja de la RM radica en una mayor resolución tisular y espacial, lo que permite distinguir las diferentes capas de la pared vesical, del tumor y mejorar la visualización de la invasión de órganos vecinos. ${ }^{1}$

Un protocolo de estudio estándar en RM consta de secuencias convencionales: T1, útil para identificar infiltración extravesical, adenopatías pélvicas y lesiones óseas; T2 sin saturación grasa, excelente para evaluar la invasión de la capa muscular. ${ }^{1}$ En algunos estudios se ha informado que la precisión de la RM solo con secuencia T2 para la estadificación, es muy baja y variable (15\% al 58\%) para enfermedad órgano confinada. ${ }^{5}$

En los últimos años, se han añadido nuevas secuencias de RM que aportan al estudio morfológico una valoración funcional y molecular, lo que ha mejorado la aproximación diagnóstica al $\mathrm{CV}^{5}$ :

- Dynamic contrast enhanced (DCE-RM): Estudio funcional con precisión en la estadificación local mayor que la secuencia T2, variando entre el $84 \%$ al $87 \% .^{5,17}$ Es además, la secuencia más útil para la diferenciación entre cambios inflamatorios postbiopsia y lesión tumoral. ${ }^{1}$

- Difusion Weighted (DW-RM): Aumenta la seguridad diagnóstica para la diferenciación entre tumor superficial vs invasivo (63\% vs 6\%) y órgano-confinado vs órgano-invasivo (69\% vs $15 \%$ ) respectivamente, ${ }^{1,17}$ con una sensibilidad para estadificación local del 80 al $88 \%$ y una especificidad del 76 al $85 \%$ cuando se combina con imágenes ponderadas en $\mathrm{T} 2^{5,18}$

Las desventajas de la RM radican en su elevado costo, menor disponibilidad, mayor susceptibilidad a variabilidades entre pacientes y la asociación entre la administración de gadolinioy el posterior desarrollo de fibrosis sistémica nefrogénica, en pacientes con nefropatía preexistente. ${ }^{1}$

\section{Impacto pronóstico del estadio clínico}

La seguridad diagnóstica en la estadificación local con TAC es motivo de discusión, existiendo datos muy discordantes en la bibliografía con tasas de exactitud global que oscilan entre $49 \%$ y $63 \%$, valores de infraestadificación del $6 \%$ al $30 \%$, sobreestadificación del $17 \%$ al 37\% ${ }^{1,19-21}$ y tasas de error en el diagnóstico tanto del estadio tumoral T2-T3 (36\%) y ganglionar (25\%), resultando en importante discrepancia entre la estadificación prequirúrgica y postquirúrgica con hallazgos histológicos. ${ }^{1,7}$

Es importante tener en cuenta que se debe retrasar la realización de la imagen al menos 7 días luego de la RTU, con el fin de minimizar los artefactos inflamatorios, que pueden confundirse con un estadio más avanzado de la enfermedad (T3) y afectar la exactitud de la estadificación. ${ }^{16}$

\section{Estadificación del tumor primario (T)}

En cuanto a la estadificación local, los estudios confirman que la sensibilidad, especificidad, y valores predictivos de la TAC, dependen del estadio pT y es clara la limitación que tiene la imagen en la distinción de tumores Ta a T2 y en el diagnóstico de T3a. ${ }^{1,7,22}$ Eso se debe a que las capas de la pared de la vejiga y la atenuación del tumor son difíciles de diferenciar, ${ }^{23}$ al igual que los tumores del cuello vesical adyacentes a la próstata y las lesiones planas sésiles. Además, el engrosamiento difuso de la pared vesical se puede dar no solo debido al tumor, sino también por llenado insuficiente, obstrucción crónica del tracto de salida, infecciones o cambios inflamatorios que son difíciles de diferenciar. ${ }^{24,25}$

Sin embargo, el rendimiento mejora para identificar tumores que invaden macroscópicamente a través de la serosa en la grasa perivesical (T3b) y órganos adyacentes (T4), ${ }^{26}$ demostrando en varios estudios una sensibilidad que va desde el $83,3 \%$ al $89 \%$ y una especificidad desde un $63 \%$ hasta el $100 \%$ en la literatura más reciente. ${ }^{5,14,16,27}$

En un estudio donde se pretendía evaluar el papel de la TAC en el estadiaje prequirúrgico de los pacientes con CV infiltrante y su impacto en el tratamiento, se encontró una infraestadificación del 50,8\% y una sobreestadificación del $20,6 \%$, con una concordancia con la patología solo en el $36 \%$ de los casos. Además, se concluyó que la TAC para el estadiaje perivesical tiene sensibilidad más baja cuanto más avanzada es dicha afectación y por el contrario su especificidad es más elevada cuanto más avanzado localmente sea el tumor, oscilando entre $44 \%$ en los pT2 y el $94 \%$ en los pT4, con VPP de 55,6\%, siendo un superior en estadios bajos y un VPN global de 64,5\%, siendo mayor en el estadio pT4 (86,7\%). Sólo en 5 de 63 casos (7,9\%), la TAC previa modificó la actitud quirúrgica y se trató de casos muy avanzados (pT3-4), ${ }^{7}$ lo cual se correlaciona con otros estudios que informan que el cambio en la conducta quirúrgica por los hallazgos de la TAC, está entre el $2 \%$ y el $5 \%{ }^{28}$

La RM tiene una seguridad similar a la TAC en la detección de CV (90\%-95\%). Su ventaja radica en la estadificación local, ${ }^{1,29}$ siendo superior para diferenciar los tumores superficiales (T1) de los MI (T2b), aunque los tumores mínimamente invasivos (T2a) todavía son difíciles de detectar con cualquiera de las modalidades. ${ }^{5}$

La diferenciación entre los tumores T2a y T2b puede no ser crítica, ya que los resultados son similares en cuanto a la supervivencia libre de recurrencia. ${ }^{30}$ Sin embargo, los tumores T2b tienen mayor tasa de metástasis ganglionar $\mathrm{y}$ es justamente ese factor, en lugar del grado de invasión muscular (T2a vs T2b), el que determina principalmente la supervivencia libre de enfermedad luego de la cistectomía. 1,31

En la valoración local con RMN se estima una sobreestadificación del 32\% al 49\% y una infraestadificación del 13\%. La sobreestadificación puede estar justificada en algunos casos por la realización del estudio de imagen precozmente tras la RTU, la cual provoca cambios inflamatorios locales que se pueden confundir con infiltración ${ }^{29,32}$

La enfermedad microscópica extravesical (T3a) está por debajo de la resolución tanto de la RMN como de la TAC, por lo tanto, es a menudo subdiagnosticada por imágenes. ${ }^{26}$ La RMN es discretamente más sensible a expensas de una menor 
especificidad, ya que puede malinterpretar los cambios inflamatorios inducidos por tumores $\mathrm{T} 2$ o post-RTU ${ }^{1}$

El T3b puede ser diagnosticado con similar seguridad con TAC y con RM, sin embargo se ha sugerido que debido a su mayor resolución para tejidos blandos, la RMN sea más sensible que la TAC para delinear la extensión extravesical (T3b). ${ }^{33}$ Dos estudios mostraron sensibilidades $y$ especificidades del $93 \%$ y $73 \%(n=36)$ y del $100 \%$ y $94 \%$ $(\mathrm{n}=108)$ para la $\mathrm{RNM},{ }^{5,34,35}$ comparado con resultados en TAC que mostraron sensibilidades del $85 \%$ al $89 \%$ y especificidades del $63 \%$ al $95 \% .14,16$

En conclusión, el impacto de la TAC en el estadiaje clínico del $\mathrm{CV}$ infiltrante es relativamente bajo, ya que la invasión de la capa muscular (T2 o mayor) es el determinante principal del manejo y pronóstico, para lo cual la TAC tiene una seguridad diagnóstica baja, mientras que la RMN multiparamétrica, especialmente las secuencias con contraste, permite valorar de forma más segura la infiltración en profundidad de la pared vesical, demostrando los mejores valores de sensibilidad y especificidad en el diagnóstico de tumor superficial (T1) vs MI (T2) que van desde $66 \%$ a $97 \%$ y de $60 \%$ a $93 \%$ respectivamente en los diferentes estudios, con VPP de $57 \%$ a $81 \%$ y VPN de $61 \%$ a $96 \%^{1,30,36,37}$

\section{Estadificación ganglionar (N)}

En el estudio de metástasis ganglionares con TAC se utiliza básicamente el tamaño como criterio de afectación, de forma que se consideran patológicos aquellos ganglios ovalados mayores de $10 \mathrm{~mm}$ en el eje corto o redondos mayores de $8 \mathrm{~mm}$. La utilización del tamaño como único criterio diagnóstico disminuye la sensibilidad de la técnica (30\%$50 \%)$, pero mantiene una alta especificidad $(80 \%-100 \%)$ con falsos positivos alrededor de $16 \%$ y falsos negativos entre $25 \%$ y $40 \% .16,19,22,38,39$

En uno de los estudios se pretendía evaluar el papel de la TAC en el estadiaje prequirúrgico. Se realizó linfadenectomía ilioobturatriz bilateral a 46 pacientes ( $73 \%$ de los casos), de los cuales la TAC infraestadificó 19,5\%, supraestadificó 21,5\% y hubo concordancia en los resultados en el $59 \%$, aunque ese porcentaje depende casi exclusivamente de los pacientes con ganglios negativos (NO), lo cual indica que cuando no existe afectación ganglionar la TAC casi siempre será negativa (VPN del $92 \%$ ), con un número escaso de falsos positivos (9\%). Pero cuando había compromiso ganglionar (N1-N2), la estimación fue mucho peor, con una tasa de falsos negativos del $26 \%$. Finalmente todos los datos concluyeron una sensibilidad del $28 \%$, especificidad de $55 \%$, valor predictivo positivo de $68 \%$ y valor predictivo negativo de $67 \%$ de la tomografía con respecto a la estadificación ganglionar. ${ }^{7}$

La RMN convencional tiene una seguridad de $73 \%-94 \%$, similar a la TAC. ${ }^{40}$ El uso de secuencias DW-RM con ADC podría mejorar el diagnóstico, aunque aún no está establecido un valor de corte del ADC que discrimine las lesiones malignas. La precisión de la TAC y la RMN convencional, valorando solo el tamaño ganglionar, es similar, siendo 70\%-90\% en TAC y 73\%-94\% en RMN. ${ }^{1,32,38}$

La PET-CT con valoración anatómica y funcional de los ganglios muestra los mejores resultados. En estudios comparativos de PET-CT y TAC se observa un aumento en la sensibilidad ( $87 \%$ vs $66 \%$ ), VPN (92\% vs $72 \%$ ) y seguridad diagnóstica (82\% vs $60 \%) .{ }^{1,41}$ Recientes publicaciones no confirman una alta especificidad de la técnica, por lo que algunos autores consideran la PET-CT más como una guía para la extensión de la linfadenectomía. ${ }^{1,42}$

\section{Metástasis a distancia (M)}

El hígado y el pulmón son las localizaciones más frecuentes, siendo la TAC y RM técnicas igualmente válidas para su diagnóstico. ${ }^{1}$

La PET-CT tiene una mayor sensibilidad global que la TAC en el diagnóstico de metástasis a distancia (54\% vs $41 \%$ ) con especificidades similares (97\% vs $98 \%$ ). La PET sola tiene limitaciones en la valoración de metástasis renales y cerebrales por su alto metabolismo, así como en lesiones menores de $8 \mathrm{~mm}$. En la detección de metástasis óseas la RM y la PET tienen resultados comparables y son superiores a la gammagrafía ósea, con sensibilidad $94 \%$ vs $72 \%$, especificidad $90 \%$ vs $75 \%$ y exactitud diagnóstica de un $92 \%$ vs $74 \%{ }^{1}$

\section{Estrategias de seguimiento}

En pacientes con cáncer de vejiga llevados a tratamiento con intención curativa, se busca detectar recurrencia pélvica y metátasis lo más temprano posible con los estudios disponibles en la actualidad. Ese objetivo debería estar guiado por el estadio pT y pN según las guías europeas, ${ }^{36}$ las cuales para el 2007 propusieron un esquema trimestral basado en examen físico completo, uroanalisis, hemograma, ecografía de abdomen, radiografía de tórax y si tiene ganglios positivos, gamagrafía ósea y TAC, ${ }^{43}$ sin embargo, eso cambió posteriormente. ${ }^{44}$

Un estudio propone realizar el seguimiento según el estadio tumoral inicial y detenerlo a los 5 años, para continuar con vigilancia funcional, enfocado solo en la derivación urinaria y los síntomas. ${ }^{37}$ Se indica TAC y Rx de tórax a los 4 y 12 meses con el fin de detectar tempranamente la mayoría de recurrencias ( $84 \%$ en los primeros 2 años).

Bochner propuso un esquema de TACy Rx de tórax a los 3 , 6,12 y 18 meses, posteriormente seguimiento anual. Para pacientes con un estadio pT2 o menor sin compromiso ganglionar ni metastásico, el esquema es a los 3 y 12 meses y luego anual sólo hasta los 3 años. ${ }^{45}$

Soukup propuso un esquema basado en el riesgo. En el estadio pT2 sin compromiso ganglionar se debería realizar TAC y Rx de tórax a los 6, 12, 18 y 24 meses y luego, anualmente. ${ }^{46}$

\section{Conclusiones}

Existen múltiples factores determinantes del pronóstico en CV-MI, entre ellos el estadio clínico y el compromiso ganglionar metastásico. Para la estadificación clínica prequirúrgica del CV-MI, la recomendación actual de las guías clínicas es emplear de manera rutinaria la Tomografía Computarizada (TAC), sin embargo, esa técnica tiene baja 
precisión diagnostica, con limitación importante para la estadificación local ( $<\mathrm{T} 3 \mathrm{a})$; mientras que la $\mathrm{RM}$ multiparamétrica con secuencias morfológicas, funcionales y moleculares (DCE y DW), permite valorar de forma más precisa la infiltración en profundidad de la pared vesical, con mayor sensibilidad y especificidad en la diferenciación de T1 vs T2b.

Ahora se encuentran disponibles numerosas técnicas de imágenes para realizar estadificación clínica, pero consideramos que algunos pacientes seleccionados se benefician de realizar RM multiparamétrica adicional para lograr clarificar un poco más el estadio de la enfermedad antes de llegar a la cirugía.

\section{Responsabilidades Éticas}

Durante la realización de este artículo de revisión, no se realizaron experimentos que involucren personas o animales, ni se requirió evaluación por parte de algún comité de ética institucional ni la obtención de consentimiento informado por parte de persona alguna. Tampoco se utilizaron datos provenientes de historias clínicas de pacientes o tomados de centros hospitalarios para la elaboración y redacción del presente artículo.

\section{Conflictos de intereses}

Los autores declaran no tener ningún conflicto de intereses.

\section{Referencias}

1 Mesa A, Nava E, Fernández Del Valle A, Argüelles B, Menéndez-Del Llano R, Sal de Rellán S. Papel de las técnicas de imagen en el diagnóstico y seguimiento del carcinoma vesical músculo invasivo. Actas Urol Esp 2018;42(07):425-434. Doi: 10.1016/j. acuro.2017.08.001

2 Ferlay J, Steliarova-Foucher E, Lortet-Tieulent J, et al. Cancer incidence and mortality patterns in Europe: estimates for 40 countries in 2012. Eur J Cancer 2013;49(06):1374-1403. Doi: 10.1016/j.ejca.2012.12.027

3 IARC. Estimated age-standardized incidence and mortality rates (World) in 2018, Africa, Tanzania, United Republic of, males, all ages [Internet] [Consultado 3 Dic 2019]. Disponible en: https:// gco.iarc.fr/today/online-analysis-map? $\mathrm{v}=2018$ \&mode $=$ population\&mode_population

4 Burger M, Catto JWF, Dalbagni G, et al. Epidemiology and risk factors of urothelial bladder cancer. Eur Urol 2013;63(02): 234-241. Doi: 10.1016/j.eururo.2012.07.033

5 Lee $\mathrm{CH}$, Tan $\mathrm{CH}$, Faria SC, Kundra V. Role of Imaging in the Local Staging of Urothelial Carcinoma of the Bladder. AJR Am J Roentgenol 2017;208(06):1193-1205. Doi: 10.2214/AJR.16. 17114

6 Prout GR, Marshall VF. The prognosis with untreated bladder tumors. Cancer 1956;9(03):551-558. Doi: 10.1002/1097-0142 (195605/06)9:3<551:aid-cncr2820090319>3.0.co;2-2

7 Navarro Medina P, Blanco Diez A, Artiles Hernández JL, Chesa Ponce N. Relación clinico-patólogica en cáncer vesical. Actas Urol Esp 2008;32(05):502-506. Doi: 10.1016/s0210-4806(08)73874-9

8 Rafique M, Javed AA. Role of intravenous urography and transabdominal ultrasonography in the diagnosis of bladder carcinoma. Int Braz J Urol 2004;30(03):185-190, discussion 191. Doi: $10.1590 / \mathrm{s} 1677-55382004000300002$

9 Datta SN, Allen GM, Evans R, Vaughton KC, Lucas MG. Urinary tract ultrasonography in the evaluation of haematuria-a report of over 1,000 cases. Ann R Coll Surg Engl 2002;84(03):203-205
10 Nicolau C, Bunesch L, Peri L, et al. Accuracy of contrast-enhanced ultrasound in the detection of bladder cancer. Br J Radiol 2011;84 (1008):1091-1099. Doi: 10.1259/bjr/43400531

11 Yousem DM, Gatewood OM, Goldman SM, Marshall FF. Synchronous and metachronous transitional cell carcinoma of the urinary tract: prevalence, incidence, and radiographic detection. Radiology 1988;167(03):613-618. Doi: 10.1148/ radiology.167.3.3363119

12 Browne RFJ, Meehan CP, Colville J, Power R, Torreggiani WC. Transitional cell carcinoma of the upper urinary tract: spectrum of imaging findings. Radiographics 2005;25(06): 1609-1627. Doi: 10.1148/rg.256045517

13 Raman SP, Fishman EK. Bladder malignancies on CT: the underrated role of CT in diagnosis. AJR Am J Roentgenol 2014; 203(02):347-354. Doi: 10.2214/AJR.13.12021

14 Sadow CA, Silverman SG, O'Leary MP, Signorovitch JE. Bladder cancer detection with CT urography in an Academic Medical Center. Radiology 2008;249(01):195-202. Doi: 10.1148/radiol. 2491071860

15 Jinzaki M, Tanimoto A, Shinmoto $\mathrm{H}$, et al. Detection of bladder tumors with dynamic contrast-enhanced MDCT. AJR Am J Roentgenol 2007;188(04):913-918. Doi: 10.2214/AJR.06.0511

16 Kim JK, Park SY, Ahn HJ, Kim CS, Cho KS. Bladder cancer: analysis of multi-detector row helical CT enhancement pattern and accuracy in tumor detection and perivesical staging. Radiology 2004;231(03):725-731. Doi: 10.1148/radiol.2313021253

17 El-Assmy A, Abou-El-Ghar ME, Mosbah A, et al. Bladder tumour staging: comparison of diffusion- and T2-weighted MR imaging. Eur Radiol 2009;19(07):1575-1581. Doi: 10.1007/s00330-0091340-7

18 Kobayashi S, Koga F, Yoshida S, et al. Diagnostic performance of diffusion-weighted magnetic resonance imaging in bladder cancer: potential utility of apparent diffusion coefficient values as a biomarker to predict clinical aggressiveness. Eur Radiol 2011; 21(10):2178-2186. Doi: 10.1007/s00330-011-2174-7

19 Baltaci S, Resorlu B, Yagci C, Turkolmez K, Gogus C, Beduk Y. Computerized tomography for detecting perivesical infiltration and lymph node metastasis in invasive bladder carcinoma. Urol Int 2008;81(04):399-402. Doi: 10.1159/000167836

20 Vargas HA, Akin O, Schöder H, et al. Prospective evaluation of MRI, ${ }^{11} \mathrm{C}$-acetate PET/CT and contrast-enhanced CT for staging of bladder cancer. Eur J Radiol 2012;81(12):4131-4137. Doi: 10.1016/j.ejrad.2012.06.010

21 Tritschler S, Mosler C, Tilki D, Buchner A, Stief C, Graser A. Interobserver variability limits exact preoperative staging by computed tomography in bladder cancer. Urology 2012;79(06): 1317-1321. Doi: 10.1016/j.urology.2012.01.040

22 Tritschler S, Mosler C, Straub J, et al. Staging of muscle-invasive bladder cancer: can computerized tomography help us to decide on local treatment? World J Urol 2012;30(06):827-831. Doi: 10.1007/s00345-011-0817-6

$23 \mathrm{Ng}$ CS. Radiologic diagnosis and staging of renal and bladder cancer. Semin Roentgenol 2006;41(02):121-138. Doi: 10.1053/j. ro.2006.02.005

24 Maurer T, Horn T, Heck M, Gschwend JE, Eiber M, Beer AJ. Current Staging Procedures in Urinary Bladder Cancer. Diagnostics (Basel) 2013;3(03):315-324. Doi: 10.3390/diagnostics3030315

25 Verma S, Rajesh A, Prasad SR, et al. Urinary bladder cancer: role of MR imaging. Radiographics 2012;32(02):371-387. Doi: 10.1148/ rg.322115125

26 Vikram R, Sandler CM, Ng CS. Imaging and staging of transitional cell carcinoma: part 1, lower urinary tract. AJR Am J Roentgenol 2009;192(06):1481-1487. Doi: 10.2214/AJR.08.1318

$27 \mathrm{Oz}$ II, Altinbas NK, Serifoglu I, Oz EB, Yagci C. The Role of Computerized Tomography in the Assessment of Perivesical Invasion in Bladder Cancer. Pol J Radiol 2016;81:281-287. Doi: $10.12659 /$ PJR.896752 
28 Blanco Díez A, Ruibal Moldes M, Suárez Pascual G, et al. Estadiaje del cáncer de vejiga infiltrante. Papel de la T.A.C. Arch Esp Urol 2003;56(01):23-29

29 Tekes A, Kamel I, Imam K, et al. Dynamic MRI of bladder cancer: evaluation of staging accuracy. AJR Am J Roentgenol 2005;184 (01):121-127. Doi: 10.2214/ajr.184.1.01840121

30 Boudreaux KJ Jr, Clark PE, Lowrance WT, et al. Comparison of american joint committee on cancer pathological stage T2a versus T2b urothelial carcinoma: analysis of patient outcomes in organ confined bladder cancer. J Urol 2009;181(02):540-545, discussion 546. Doi: 10.1016/j.juro.2008.10.038

31 Yu RJ, Stein JP, Cai J, Miranda G, Groshen S, Skinner DG. Superficial (pT2a) and deep (pT2b) muscle invasion in pathological staging of bladder cancer following radical cystectomy. J Urol 2006;176(02):493-498, discussion 498-499. Doi: $10.1016 /$ j.juro.2006.03.065

32 Liedberg F, Bendahl PO, Davidsson T, et al. Preoperative staging of locally advanced bladder cancer before radical cystectomy using 3 tesla magnetic resonance imaging with a standardized protocol. Scand J Urol 2013;47(02):108-112. Doi: 10.3109/00365599.2012.721394

33 McKibben MJ, Woods ME. Preoperative imaging for staging bladder cancer. Curr Urol Rep 2015;16(04):22. Doi: 10.1007/ s11934-015-0496-8

34 Kim B, Semelka RC, Ascher SM, Chalpin DB, Carroll PR, Hricak H. Bladder tumor staging: comparison of contrast-enhanced CT, T1- and T2-weighted MR imaging, dynamic gadolinium-enhanced imaging, and late gadolinium-enhanced imaging. Radiology 1994; 193(01):239-245. Doi: 10.1148/radiology.193.1.8090898

35 Ghafoori M, Shakiba M, Ghiasi A, Asvadi N, Hosseini K, Alavi M. Value of MRI in local staging of bladder cancer. Urol J 2013;10(02): 866-872

36 Witjes JA, Bruins HM, Cathomas R, Compérat E, Cowan NC, Gakis G, Hernández V, Lorch A, Ribal MJ, et al. Muscle-invasive and Metastatic Bladder Cancer Guidelines 2020 [Internet]. EUA 2020 [Consultado marzo 2020]. Disponible en: uroweb.org/guideline/ bladder-cancer-muscle-invasive-and-metastatic/
37 Nieuwenhuijzen JA, de Vries RR, van Tinteren H, et al. Follow-up after cystectomy: regularly scheduled, risk adjusted, or symptom guided? Patterns of recurrence, relapse presentation, and survival after cystectomy. Eur J Surg Oncol 2014;40(12):1677-1685. Doi: 10.1016/j.ejso.2013.12.017

38 Paik ML, Scolieri MJ, Brown SL, Spirnak JP, Resnick MI. Limitations of computerized tomography in staging invasive bladder cancer before radical cystectomy. J Urol 2000;163(06):1693-1696

39 Lodde M, Lacombe L, Friede J, Morin F, Saourine A, Fradet Y. Evaluation of fluorodeoxyglucose positron-emission tomography with computed tomography for staging of urothelial carcinoma. BJU Int 2010;106(05):658-663. Doi: 10.1111/j.1464-410X.2010.09212.x

40 Turker P, Bostrom PJ, Wroclawski ML, et al. Upstaging of urothelial cancer at the time of radical cystectomy: factors associated with upstaging and its effect on outcome. BJU Int 2012;110(06): 804-811. Doi: 10.1111/j.1464-410X.2012.10939.x

41 Chakraborty D, Mittal BR, Kashyap R, et al. Role of fluorodeoxyglucose positron emission tomography/computed tomography in diagnostic evaluation of carcinoma urinary bladder: comparison with computed tomography. World J Nucl Med 2014;13(01):34-39. Doi: 10.4103/1450-1147.138572

42 Swinnen G, Maes A, Pottel H, et al. FDG-PET/CT for the preoperative lymph node staging of invasive bladder cancer. Eur Urol 2010;57 (04):641-647. Doi: 10.1016/j.eururo.2009.05.014

43 Stenzl A, Cowan NC, De Santis M, et al. Guidelines on Bladder Cancer Muscle-invasive and Metastatic. Eur Urol 2008

44 Stenzl A, Cowan NC, De Santis M, et al. The updated EAU guidelines on muscle-invasive and metastatic bladder cancer. Eur Urol 2009; 55(04):815-825. Doi: 10.1016/j.eururo.2009.01.002

45 Bochner BH, Montie JE, Lee CT. Follow-up strategies and management of recurrence in urologic oncology bladder cancer: invasive bladder cancer. Urol Clin North Am 2003;30 (04):777-789. Doi: 10.1016/s0094-0143(03)00061-2

46 Soukup V, Babjuk M, Bellmunt J, et al. Follow-up after surgical treatment of bladder cancer: a critical analysis of the literature. Eur Urol 2012;62(02):290-302. Doi: 10.1016/j.eururo.2012.05.008 